\title{
Development of a EST dataset and characterization of EST-SSRs in a traditional Chinese medicinal plant, Epimedium sagittatum (Sieb. Et Zucc.) Maxim
}

Shaohua Zeng ${ }^{1,2+}$, Gong Xiao ${ }^{1,2+}$, Juan Guo ${ }^{1,2}$, Zhangjun Fei ${ }^{3,4}$, Yanqin Xu', Bruce A Roe ${ }^{5}$, Ying Wang ${ }^{1 *}$

\begin{abstract}
Background: Epimedium sagittatum (Sieb. Et Zucc.) Maxim, a traditional Chinese medicinal plant species, has been used extensively as genuine medicinal materials. Certain Epimedium species are endangered due to commercial overexploition, while sustainable application studies, conservation genetics, systematics, and marker-assisted selection (MAS) of Epimedium is less-studied due to the lack of molecular markers. Here, we report a set of expressed sequence tags (ESTs) and simple sequence repeats (SSRs) identified in these ESTs for E. sagittatum.

Results: CDNAs of E. sagittatum are sequenced using 454 GS-FLX pyrosequencing technology. The raw reads are cleaned and assembled into a total of 76,459 consensus sequences comprising of 17,231 contigs and 59,228 singlets. About 38.5\% $(29,466)$ of the consensus sequences significantly match to the non-redundant protein database (E-value < 1e-10), 22,295 of which are further annotated using Gene Ontology (GO) terms. A total of 2,810 EST-SSRs is identified from the Epimedium EST dataset. Trinucleotide SSR is the dominant repeat type (55.2\%) followed by dinucleotide (30.4\%), tetranuleotide (7.3\%), hexanucleotide (4.9\%), and pentanucleotide (2.2\%) SSR. The dominant repeat motif is AAG/CTT (23.6\%) followed by AG/CT (19.3\%), ACC/GGT (11.1\%), AT/AT (7.5\%), and AAC/ GTT (5.9\%). Thirty-two SSR-ESTs are randomly selected and primer pairs are synthesized for testing the transferability across 52 Epimedium species. Eighteen primer pairs (85.7\%) could be successfully transferred to Epimedium species and sixteen of those show high genetic diversity with 0.35 of observed heterozygosity $(\mathrm{Ho})$ and 0.65 of expected heterozygosity (He) and high number of alleles per locus (11.9).

Conclusion: A large EST dataset with a total of 76,459 consensus sequences is generated, aiming to provide sequence information for deciphering secondary metabolism, especially for flavonoid pathway in Epimedium. A total of 2,810 EST-SSRs is identified from EST dataset and 1580 EST-SSR markers are transferable. E. sagittatum ESTSSR transferability to the major Epimedium germplasm is up to $85.7 \%$. Therefore, this EST dataset and EST-SSRs will be a powerful resource for further studies such as taxonomy, molecular breeding, genetics, genomics, and secondary metabolism in Epimedium species.
\end{abstract}

\section{Background}

Herb epimedii, a traditional Chinese medicinal herb, is prepared from the aerial parts of Epimedium species of Berberidaceae, which is a basal eudicot family containing enormous medicinal plants. E. sagittatum (Sieb. Et

\footnotetext{
* Correspondence: yingwang@wbgcas.cn

† Contributed equally

${ }^{1}$ Key Laboratory of Pant Germplasm Enhancement and Speciality Agriculture, Wuhan Botanical Garden, Chinese Academy of Sciences, Wuhan, Hubei 430074, China
}

(c) 2010 Zeng et al; licensee BioMed Central Ltd. This is an Open Access article distributed under the terms of the Creative Commons Attribution License (http://creativecommons.org/licenses/by/2.0), which permits unrestricted use, distribution, and reproduction in any medium, provided the original work is properly cited. cies, E. brevicornu Maxim, E. pubescens Maxim, E. wushanense T. S. Ying, and E. koreanum Nakai, is listed in Chinese Pharmacopoeia [1]. Herb epimediicontains various bioactive components and has been utilized extensively as a tonic and antirheumatic medicinal herb for thousands of years in China. Currently, Herb epimedii is still widely used to treat many diseases such as impotence, frequency/urgency of urination, coronary heart disease, chronic bronchitis, and neurasthenia [2]. In 
addition, Epimedium species are also used as ground cover plants and ornamental plants because of the abundance of flower colours and flower patterns. Therefore, Epimedium species possess enormous potentials for commercial application. The molecular research of Epimedium, however, is lagged behind. Firstly, at present, many studies focus on extracting bioactive components from Epimedium [3-5], but metabolic pathways involved in producing these bioactive components are still largely unknown. Secondly, many studies have attempted to distinguish taxonomically Epimedium species and detect the genetic diversity of Epimedium [6-8]. But only a few of genomic markers have been exploited, so far, in our lab for studying genetic diversity of natural populations, including 14, 19, and 12 SSRs for E. sagittatum [9], E. koreanum [10], and E. brevicornu [11], respectively. The most important thing is that, according to our knowledge, most genomic SSRs exploited fail to transfer in other Epimedium species. Thus, more markers are needed for in-depth understanding of the natural diversity and for developing strategies for sustainable utilization of Epimedium. Thirdly, although many papers reported taxonomic results of Epimedium using different molecular markers such as RAPD [12], RFLP [12], AFLP $[13]$, and rDNA $[6,7,14]$, the taxonomical relationships among Epimedium species, especially closely related Chinese species, are still not clear because of the shortcoming of molecular markers with little polymorphic information or the limited number of species sampled in former studies. Moreover, the morphological characteristics of E. sagittatum vary greatly among different populations, and it is still not known whether it belongs to polytypic species or a complex of cryptic species. Finally, according to our knowledge, the novel gene discovery and molecular breeding of Epimedium new cultivars haven't been started yet due to the lack of genetic and genomic information.

Generation of a large-scale expressed sequence tag (EST) dataset is a useful approach to accelerate the researches of non-model species, especially for Epimedium. As a valuable resource for comparative genomics, functional genomics and biodiversity study, EST database has been established for model plant species and non-model species (citrus, grape, kiwifruit, etc.). So far, only three Ranunculales species (Aquilegia formosa $\times$ Aquilegia pubescens, Eschscholzia californica, and Papaver somniferum) have EST sequences available in the public databases and very limited number of EST sequences are available for these species. Although two cDNA libraries have been constructed using flower and leaf materials of E. brevicornu [15,16], no EST sequences have been uploaded to National Center for Biotechnology Information (NCBI, http:// www.ncbi.nlm.nih.gov) database yet. As of June 2009, only 398 nucleotide sequences are available for Epimedium in NCBI.

Comparing with other types of molecular markers, SSR marker has many advantages including high abundance, random distribution in the entire genome, high information content, codominant inheritance, and reproducibility. According to the original sequences used for identification of simple repeats, SSRs are divided in two categories, genomic SSRs derived from random genomic sequences and EST-SSRs derived from expressed sequence tags. It is very time-consuming, cost-expensive and labor-intensive for developing genomic SSRs from the repeat-enriched genomic library when compared with the development of EST-SSR. Genomic SSRs have neither genic function nor close linkage to transcriptional regions, while EST-SSRs are potentially tightly linked with functional genes that perhaps control certain important agronomic characters [17]. Therefore, ESTSSRs are very useful for MAS in plant breeding. Besides, EST-SSR markers contain high transferability because EST-SSRs are derived from expressed sequences that are more conserved than the non-genic sequences and can be found in other relative species [18]. Due to the sharp increase of EST sequences deposited in NCBI, more and more EST-SSRs have been identified and used extensively for comparative mapping, DNA fingerprinting, biodiversity, and evolutionary studies in lots of species $[19,20]$. So far, surveying the polymorphism, diversity and transferability of EST-SSRs have been performed in Vitis [18], Citrus [20], Coffea [21,22], Medicago [23], Glycine [24], Phaseolus [25], Hordeum [26], Festuca [27], and Triticum [28].

Here, we report the generation of a large-scale EST dataset from young leaves of E. sagittatum using the cost-effective 454 GS-FLX pyrosequencing technology, the development and characterization of a set of ESTSSRs, and the transferability of EST-SSRs across genus Epimedium. Therefore, the EST dataset can lay a foundation and facilitate MAS breeding and taxonomy in genus Epimedium, and decode secondary metabolism in Epimedium species.

\section{Results}

\section{Contig assembly of 454 reads}

A total of 228,768 sequences $(51.7 \mathrm{Mb})$ are generated from leaf cDNAsof E. sagittatum. After removing SMART primers, polyA tail, and low quality sequences, a total of 217,380 high quality sequences is retained with a total length of $50.9 \mathrm{Mb}$ and an average length of 224.9 bp ranging from $50 \mathrm{bp}$ to $556 \mathrm{bp}$ (Additional file 1 ). After clustering and assembly, 76,459 consensus sequences with a total length of $18.8 \mathrm{Mb}(6.5 \mathrm{Mb}$ of 17,231 contigs and $12.3 \mathrm{Mb}$ of 59,228 singlets) are obtained (Additional file 1). The length of contigs ranges 
from $51 \mathrm{bp}$ to $1,911 \mathrm{bp}$ with an average of $375.9 \mathrm{bp}$, and that of singlets ranges from $50 \mathrm{bp}$ to $556 \mathrm{bp}$ with an average of 207.8 bp (Additional file 1).

The size distribution of Epimedium consensuses is shown in table 1 . The length for the majority of ESTs is 201-300 bp, which is consistent with the 454 GS-FLX sequencing capacity. After the assembly, although the size distribution pattern of singlets is similar to that of whole EST dataset, there are 5,497 contigs with sequences longer than 400 bp (Table 1 ). In addition, about $7.2 \%$ of contigs are still less than $200 \mathrm{bp}$ after assembly. This might be due to the short length of the individual read and/or the low coverage of the transcriptome represented in this dataset. Most of the consensuses are derived from few reads. For example, 7,509 (43.6\% out of contigs) and 3,074 (17.8\% out of contigs) consensuses are derived from 2 and 3 reads, respectively (Table 2).

\section{Structural and functional annotation of ESTs}

The consensus sequences are blasted against non-redundant protein database (NR database) and 29,466 consensuses comprising of 10,245 contigs and 19,221 singlets have significant hits to NR database (Additional file 1). All the consensus sequences are also compared against Pfam domain database and about $50 \%(38,220)$ of them are successfully annotated. Gene Ontology (GO) terms are further assigned to 38,220 Epimedium consensus sequences that are successfully annotated based on Pfam matches. Only 22,295 Epimedium consensus sequences are able to be assigned GO numbers. Figure 1 shows the percentage distributions of gene ontology terms according to the GO consortium. Protein metabolic process $(23.4 \%)$ is the most dominant group out of 12,242 consensus sequences that are annotated to the biological process category. It is followed by the biosynthetic process at $23.2 \%$, photosynthesis at $22.5 \%$, generation of precursor metabolites and energy at 19.3\%, cellular process at $15.7 \%$, translation at $13.6 \%$, metabolic process at $10.5 \%$, and carbohydrate metabolic process at 9.3\% (Figure 1A). A total of 13,807 sequences could be assigned to the molecular function category. Binding $(30.5 \%)$ is the most dominant group followed by catalytic activity (20.6\%), structural molecular activity (12.7\%), hydrolase activity (11.9\%), nucleotide binding (10.5\%), transferase activity (6.9\%), and transcription regulator
Table 2 Number of ESTs in the assembled consensus sequences

\begin{tabular}{cc}
\hline Number of reads per consensus & Number of consensuses \\
\hline 2 & $7,509(43.6 \%)$ \\
3 & $3,074(17.8)$ \\
4 & $1,600(9.3 \%)$ \\
5 & $1,029(6.0 \%)$ \\
6 & $667(3.9 \%)$ \\
7 & $499(2.9 \%)$ \\
8 & $385(2.2 \%)$ \\
9 & $267(1.5 \%)$ \\
10 & $192(1.1 \%)$ \\
$11-15$ & $688(4.0 \%)$ \\
$16-20$ & $352(2.0 \%)$ \\
$21-25$ & $204(1.1 \%)$ \\
$26-30$ & $138(0.8 \%)$ \\
$31-35$ & $94(0.5 \%)$ \\
$36-40$ & $81(0.5 \%)$ \\
$41-45$ & $60(0.3 \%)$ \\
$46-50$ & $43(0.2 \%)$ \\
$>51$ & $349(2.0 \%)$ \\
\hline
\end{tabular}

Note: the numbers in parentheses indicate the percentages of certain consensuses in all contigs.

activity (2.2\%) (Figure 1B). With regard to the cellular component, $64.9 \%$ of 12,308 consensus sequences are assigned to membrane followed by intracellular (15.1\%), ribosome $(12.0 \%)$ and extracellular region (5.8\%) (Figure 1C).

Being famous medicinal plants used in China, Epimedium species have flavonoids as the major phytochemical components. There are also many other unidentified compounds in Epimedium species [29]. Thus, genes related to secondary metabolism, especially for the flavonoid biosynthesis, are under further studies in order to decipher the molecular mechanism of natural variation of phytochemical components and facilitate the molecular breeding of medicinal cultivars. In our EST collection, 29 EST consensus sequences are annotated relating to secondary metabolic process (Figure 1A), including genes encoding key enzymes in the flavonoid biosynthetic pathway such as phenylalanine ammonia-lyase, cinnamate-4-hydroxylase, 4-counaroyl:ligase CoA, chalcone synthase, chalcone isomerase, flavanone 3 hydroxylase, flavanone 3' hydroxylase, flavanone 3'5' hydroxylase, flavonol synthase, flavone synthase,

Table 1 Size distribution of E. sagittatum ESTs before and after assembly

\begin{tabular}{|c|c|c|c|c|c|}
\hline Items & $1-100 \mathrm{bp}$ & $101-200 \mathrm{bp}$ & 201-300 bp & $301-400 \mathrm{bp}$ & $>400 \mathrm{bp}$ \\
\hline Cleaned EST sequence & 17,801 & 50,516 & 147,708 & 1,334 & 21 \\
\hline Singlet & 6,720 & 14,171 & 37,444 & 875 & 18 \\
\hline Contig & 163 & 1,084 & 5,461 & 5,026 & 5,497 \\
\hline Consensus & 6,883 & 15,255 & 42,905 & 5,901 & 5,515 \\
\hline
\end{tabular}




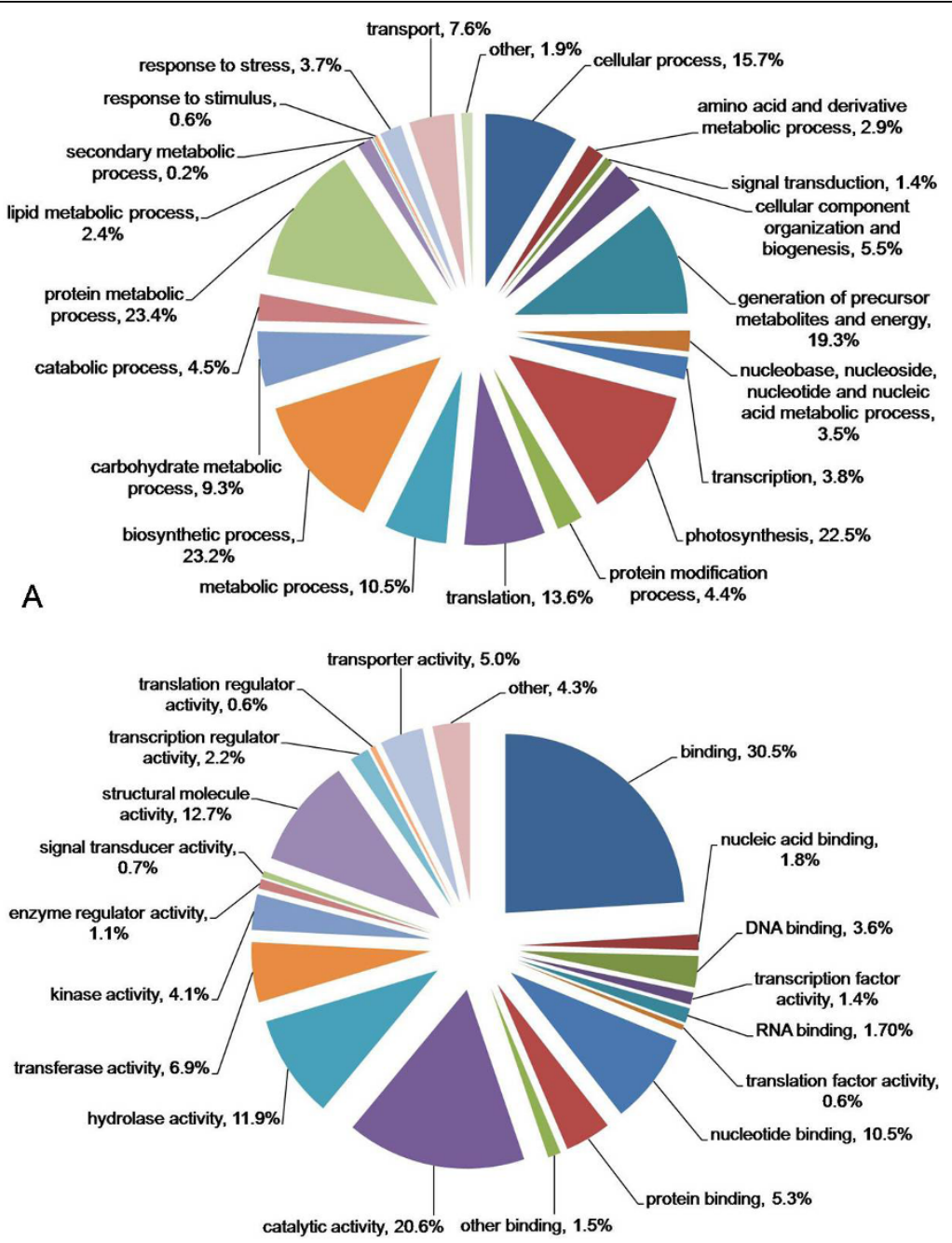

B

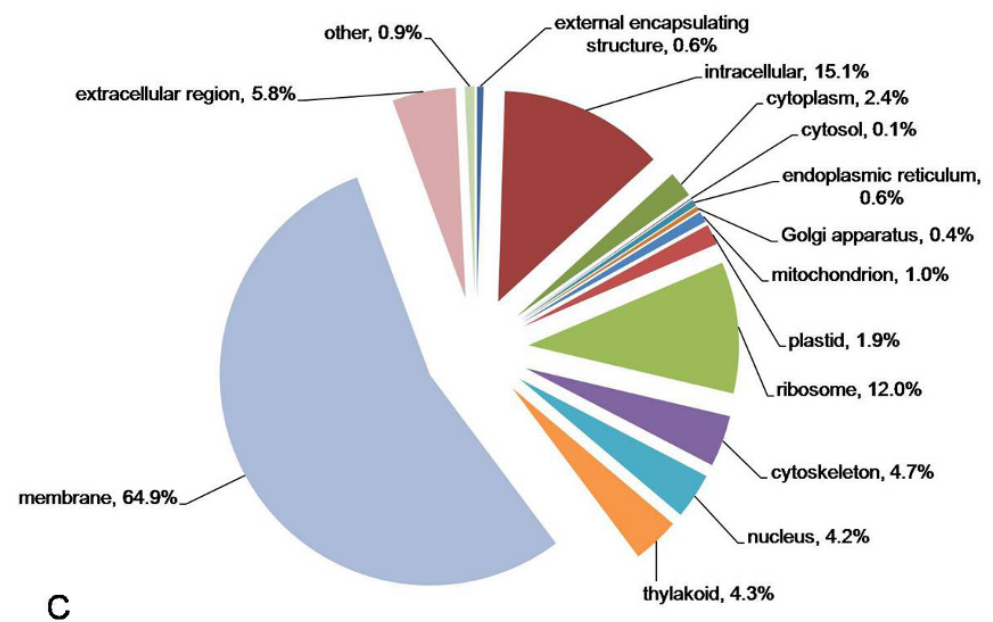

Figure 1 Pie chart representations of GO-annotation results of 22,295 E. sagittatum consensus sequences. The total numbers of Epimedium consensus sequences annotated for each category are 12,242 for Biological Process (A), 13,807 for Molecular Function (B), and 12,308 for Cellular Component (C). Since one gene product can be assigned to more than one GO terms, the total percentage in each category could excess $100 \%$. 
dihydroflavonol 4-reductase, anthocyanidin synthase, rhamnosyl transferase, and UDPG-flavonoid glucosyl transferase. In addition, more than 300 EST consensus sequences were annotated relating to transcription regulator activity (Figure 1B), part of which might play roles in regulating flavonoid biosynthesis and metabolism. For example, regulatory genes are annotated as members of WD40 family, MYB family, and bHLH family that can form a triplex compound to regulate the specific expression patterns of flavonoid structural genes. All these structural and regulatory genes are being cloned and functional studies are carrying out in our lab. Further studies such as molecular modification (or gene transformation) and metabolic engineering of enzymes are also in progress.

\section{Frequency and distribution of EST-SSRs in the Epimedium transcriptome}

A total of 2,629 sequences containing 2,810 SSRs have been identified from 76,459 consensus sequences, with 167 EST sequences containing at less two SSRs. The frequency of EST-SSRs observed in Epimedium transcriptome is $3.67 \%$, and the distribution density is 149.5 per $\mathrm{Mb}$. The occurrence of different repeat type is shown in Table 3. The most abundant repeat type is trinucleotide $(55.2 \%, 1,552)$ followed by dinucleotide $(30.4 \%, 855)$, tetranucleotide $(7.3 \%, 204)$, hexanucleotide $(4.9 \%, 137)$, and pentanucleotide repeat unit $(2.2 \%, 62)$. The frequencies of EST-SSRs distributed in different repeat numbers are also shown in Table 3 . There are 1,063 SSRs with 5 tandem repeats, which is the most common repeat number (37.8\%) followed by 6 tandem repeats $(21.2 \%, 595), 4$ tandem repeats $(11.9 \%, 333), 7$ tandem repeats $(9.8 \%$, $274)$ and 8 tandem repeats $(7.5 \%, 210)$. The dominant repeat motif is AAG/CTT with a frequency of $23.6 \%$ (663), followed by AG/CT (19.3\%, 543), ACC/GGT (11.1\%, 312), AT/AT (7.5\%, 211), and AAC/GTT (5.9\%, 165) (Table 4). More details about different repeat motif of di- and trinucleotide repeats in EST-SSRs are listed in table 4 . It is interesting that there is no CG/GC

Table 3 Frequencies of repeat type with repeat numbers in EST-SSRs from E. sagittatum

\begin{tabular}{ccccccccccc}
\hline Motif length & \multicolumn{1}{c}{ Repeat number } & \multicolumn{3}{c}{ total } & $\%$ \\
\cline { 2 - 9 } & $\mathbf{4}$ & $\mathbf{5}$ & $\mathbf{6}$ & $\mathbf{7}$ & $\mathbf{8}$ & $\mathbf{9}$ & $\mathbf{1 0}$ & $\mathbf{1 0}$ & & \\
\hline Di & - & - & 234 & 171 & 147 & 78 & 58 & 167 & 855 & 30.4 \\
Tri & - & 1,010 & 353 & 99 & 58 & 11 & 12 & 9 & 1,552 & 55.2 \\
Tetra & 156 & 41 & 4 & 1 & 2 & 0 & 0 & 0 & 204 & 7.3 \\
Penta & 53 & 5 & 1 & 1 & 2 & 0 & 0 & 0 & 62 & 2.2 \\
Hexa & 124 & 7 & 3 & 2 & 1 & 0 & 0 & 0 & 137 & 4.9 \\
total & 333 & 1,063 & 595 & 274 & 210 & 89 & 70 & 176 & 2,810 & \\
$\%$ & 11.9 & 37.8 & 21.2 & 9.8 & 7.5 & 3.2 & 2.5 & 6.3 & &
\end{tabular}

Table 4 Frequencies of different repeat motifs of di- and trinucleotide repeats in EST-SSRs from E. sagittatum

\begin{tabular}{|c|c|c|c|c|c|c|c|c|c|}
\hline \multirow[t]{2}{*}{ Repeat motif } & \multicolumn{7}{|c|}{ Repeat number } & \multirow[t]{2}{*}{ total } & \multirow[t]{2}{*}{$\%$} \\
\hline & 5 & 6 & 7 & 8 & 9 & 10 & $>10$ & & \\
\hline $\mathrm{AG} / \mathrm{CT}$ & - & 128 & 103 & 103 & 56 & 43 & 110 & 543 & 19.3 \\
\hline AT/AT & - & 47 & 52 & 33 & 15 & 11 & 53 & 211 & 7.5 \\
\hline $\mathrm{AC} / \mathrm{GT}$ & - & 59 & 16 & 11 & 7 & 4 & 4 & 101 & 3.6 \\
\hline $\mathrm{AAG} / \mathrm{CTT}$ & 384 & 171 & 47 & 39 & 7 & 9 & 6 & 663 & 23.6 \\
\hline ACC/GGT & 234 & 55 & 11 & 10 & 2 & & 0 & 312 & 11.1 \\
\hline $\mathrm{AAC/GTT}$ & 116 & 23 & 18 & 5 & 1 & 1 & 1 & 165 & 5.9 \\
\hline AGC/CGT & 58 & 25 & 9 & 1 & & & 0 & 93 & 3.3 \\
\hline ACG/CTG & 51 & 16 & 3 & 1 & & & 0 & 71 & 2.5 \\
\hline AGG/CCT & 37 & 26 & 4 & & & & 0 & 67 & 2.4 \\
\hline AAT/ATT & 47 & 14 & 3 & 1 & & & 0 & 65 & 2.3 \\
\hline ACT/ATG & 45 & 7 & 2 & 1 & & 1 & 1 & 57 & 2 \\
\hline AGT/ATC & 35 & 16 & 2 & & 1 & 1 & 1 & 56 & 2 \\
\hline $\mathrm{CCG} / \mathrm{CGG}$ & 3 & & & & & & & 3 & 0.1 \\
\hline
\end{tabular}

repeat motif and very few CCG/CGG repeats in our results.

\section{The Epimedium EST-SSR Transferability}

A total of 32 primer pairs (Additional file 2), referred to EsESP01 to EsESP32, have been stochastically selected among the 2,810 microsatellites to amplify 10 dinucleotide SSRs and 22 trinucleotide SSRs for testing the transferability in other Epimedium species (Additional file 3). Of these primer pairs, seven primer pairs (EsESP1, EsESP3, EsESP4, EsESP20, EsESP24, EsESP31, and EsESP32) cannot amplify any fragment in E. sagittatum, suggesting that these primers are not well designed. Four primers, EsESP14, EsESP19, EsESP26, and EsESP27, amplify numerous non-target bands, suggesting these primers are also problematic. Three primer pairs, EsESP05, EsESP25, and EsESP29, succeed in transferring to other Epimedium species, but amplify PCR products larger than the expected sizes. The rest of primer pairs produces polymorphic bands in most Epimedium species as shown in figure 2. Therefore, 85.7\% (18 out of 21) EST-SSR primers can be transferred successfully to other Epimedium species. In theoretically, there are $56.2 \%(18 / 32)$ of 2810 , namely $\sim 1580$, E. sagittatum EST-SSRs markers suitable for genetic studies in genus Epimedium.

Species from other genera, including Vancouveria hexandra, Nandina domestica Thunb., Berberis julianae Schneid., Berberis sargentiana Schneid., Dysosma versipellis (Hance) M. Cheng ex Ying, Mahonia bealei (Fort.) Carr., and Mahonia fortunei (Lindl.) Fedde., have been used to test the transferability of Epimedium EST-SSRs across different genus of Berberidaceae. A total of 8 primers, EsESP6, EsESP7, EsESP9, EsESP10, EsESP11, EsESP13, EsESP23, and EsESP28, can produce PCR 
fragments in $V$. hexandra (the most closely related genus reported by Wang et al [30]) with similar sizes and same SSR motifs as those in Epimedium (sequence data not shown). Only four primers, EsESP8, EsESP13, EsESP18, and EsESP21, can amplify PCR fragments in other Berberidaceae species mentioned above. However, sequences of the PCR fragments from genera Berberis, Nandina, Dysosma, and Mahonia, do not contain the SSR motifs found in Epimedium species (data not shown). Therefore, Epimedium EST-SSRs can successfully transfer to Vancouveria, but not other Berberidaceae genera tested.

\section{The genetic diversity and dendrogram in genus Epimedium}

A total of 55 individuals representing 52 species (Additional file 3) have been used to evaluate the genetic diversity in genus Epimedium. Two primer pairs, EsESP07 and EsESP08, detect a single band in some species while null in other species. Thus, they are excluded for further study and the remaining 16 ESTSSRs are used to estimate the genetic diversity among 52 species. A high level of genetic diversity in genus Epimedium is discovered (Additional file 2). The number of alleles per locus ranges from 3 to 27 with an average of 11.9 alleles. Ho ranges from 0.04 to 0.6 with an average of 0.35 . He ranges from 0.17 to 0.94 with an average of 0.65. Polymorphism information content (PIC) is from 0.17 for EsESP22 locus to 0.93 for EsESP21 locus. The observed length of PCR products vary greatly in a large spectrum compared with the fragment size in E. sagittatum (Additional file 2). The size variation of amplicons reflects the length of repetitive sequences in each SSR locus, suggesting that the polymorphism might be related to the differences of tandem repeat numbers (Figure 2).

In order to study the evolutionary relationship among Epimedium species, those 16 EST-SSR primers successfully transferring in almost all Epimedium species are used for reconstructing Epimedium phylogeny. V. hexandra is used as an outgroup species, because it is more closely related to Epimedium genus than Jeffersonia dubia (Maxim.) Benth. et Hook. f. and Podophyllum peltatum [30]. Furthermore, sequences of some loci in $V$. hexandra amplified by Epimedium EST-SSR primers confirm that similar single nucleotide repeats exist in $V$. hexandra as mentioned above. As shown in additional file 4, the phylogenetic relationship among all 52 species are supported by low bootstrap values, especially for Chinese species, partially due to the small number of EST-SSRs and the nature of EST-SSR with low PIC used in this study. Previous studies also found it is hard to differentiate the closely related Chinese species using different types of molecular markers including RAPD [12], RFLP [12], AFLP [13], and rDNA [6,7,14]. Therefore, more EST-SSR markers or combining results of different types of markers would provide more opportunities for better resolving the phylogeny among Epimedium species, which is an on-going project in our lab.

\section{Discussion}

\section{E. sagittatum ESTs annotation}

Currently, numerous studies are focusing on isolating the Epimedium bioactive components [3-5], but the potential molecular mechanism producing bioactive components is still unclear. Furthermore, little or no information regarding to its biological conservation, population genetics, molecular breeding, and molecular biology is reported for Epimedium species due to the lack of background information. The 454 GS-FLX pyrosequencing technology is a very cost-effective method to obtain large-scale EST sequences and will speed up the researches of less-studied Epimedium species. The technology has been broadly used for bacteria de novo sequencing, cDNA sequencing, small RNA sequencing, metagenomic sequencing and whole genome shotgun survey http://www.454.com/news-events/publications. asp. Margulies et al. [31] demonstrated that the technology had 100 fold sequencing capability at the cost of relative low accuracy than Sanger-based capillary electrophoresis sequencing systems when the GS20 sequencing machine was used. Recently, the new generation

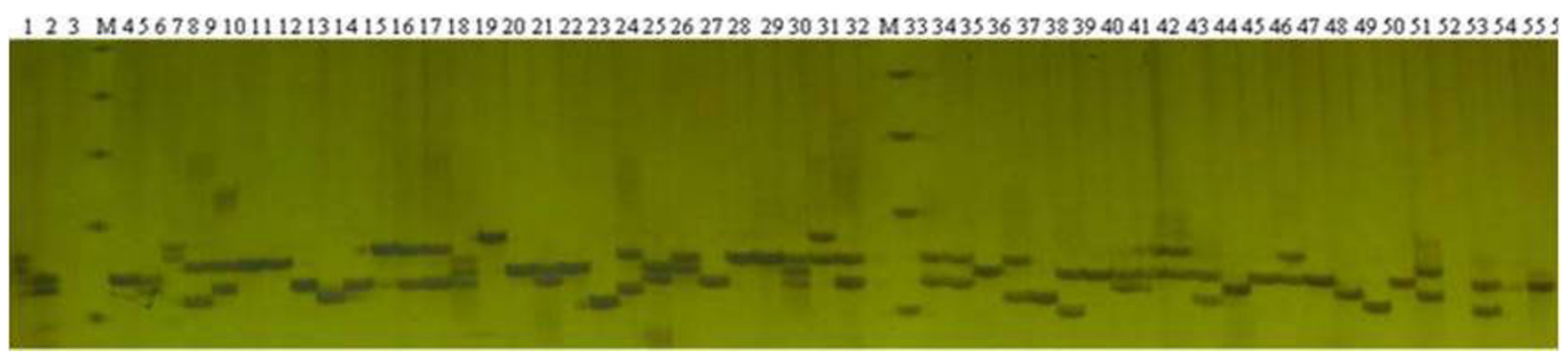

Figure 2 Polyacrylamide gel electrophoresis of PCR fragments amplified by EsESP30 primer pairs. Lane $1-55$ corresponded to the species listed in additional file 3. M indicated 25 bp ladder marker. 
machine, GS-FLX, possesses more powerful sequencing capability of up to 200-300 bp per read and higher sequencing accuracy than that of GS20 http://www.454. com. In this study, an Epimedium transcriptome population is sequenced by 454 GS-FLX system, and a total of 226,544 sequence reads are generated. After assembly, 76,459 consensus sequences are obtained.

It is noticeable that only 29,466 Epimedium consensuses have significant hits in the NR protein database (Additional file 1). Thus, there are a large number of sequences that don't have any significant match in other species $(46,993)$. This might be due to the following reasons: (1) the shortness of sequence reads leading to assemble difficultly, (2) inefficiency of blast using short sequences and the incompleteness of the known database, (3) the advantages of deep sequencing, which can discover novel genes with low expression levels, (4) the shortness of sequence reads resulting in low efficiency of annotation, which was confirmed by correlation test between sequence length and percentage of sequences successfully annotated (Additional file 5), and (5) part of consensuses representing UTRs. With regard to the first point, the size of raw reads ranged from 50 to $556 \mathrm{bp}$ with an average length of $224.9 \mathrm{bp}$. After assembly, most contigs still range from 201 to $300 \mathrm{bp}$, and the average length only increased up to $245.7 \mathrm{bp}$. It is also supported by the fact that most contigs were assembled with 2-5 reads (Table 2). Thus, the shortness of sequences is the main disadvantage in this scenario when using 454 pyrosequencing technologies. Concerning on the second point, short query sequences might not be able to have sequence matches with a significant low E-value and high score, thus resulting in a high level of false negative results. In addition, Ranunculales is much less studied when compared with core eudicots and monocots, suggesting that a lot of Ranunculales lineage-specific genes might not be included in the database. As to the third point, EST fragments are directly sequenced instead of being cloned and sequenced, which leads to the truly random sequencing of all the expressed genes and might facilitate the discovery of rare genes. Emrich et al [32] found that coupling of LCM (Laser Capture Microdissection) and 454 sequencing technologies could facilitate the discovery of rare, possibly cell-type-specific transcripts. In summary, the nature of shortness in reads generated by 454 GS-FLX sequencing technology is the origin of low efficiency of annotation. However, it is still possible that new genes and/or Ranunculales- or even Epimedium-specific novel sequences could be discovered due to large-scale sequencing.

\section{Potential applications of Epimedium EST dataset}

Combining all the available information, we have a set of 22,295 GO-annotated Epimedium consensus sequences, which is very valuable and cost-effective for a non-model organism by sequencing one tissue at certain developmental stage. Additionally, 10.5\%, 9.3\%, 3.0\%, and $0.2 \%$ of 12,242 Epimedium GO-annotated consensuses for biological processes are related to metabolic process, carbohydrate metabolic process, amino acid and derivative metabolic process, and secondary metabolic process, respectively (Figure 1A). Many bioactive components of Epimedium might derive from these biological processes (Figure 1A). A total of 13,807 Epimedium consensuses is annotated for molecular function, $2.2 \%$ and $20.6 \%$ of which have transcription regulator activity and catalytic activity, respectively, suggesting that these genes might take part in regulating and catalyzing the production of bioactive components (Figure 1B). All of these sequences can provide fundamental information for the metabolic-engineering and molecular breeding of Epimedium.

In details, this large EST dataset of Epimedium is a powerful resource for further studies. Firstly, it can facilitate cloning genes of interest, especially for genes involved in controlling metabolic pathways such as the flavonoid pathway. The Epimedium EST dataset generated in our study will provide enough sequence information for designing primers to obtain full-length genes. Therefore, these sequences will promote the molecular study of secondary metabolism in medicinal Epimedium plants. Secondly, molecular markers can be exploited based on EST sequences for researches in the fields of biodiversity, taxonomy, and population genetics. Although genomic SSRs have been developed from Epimedium, they have much lower transferability among different Epimedium species than EST-SSRs [9-11]. Therefore, EST-SSRs can further promote the taxonomic study of Epimedium species, especially those closely related Chinese species. Thirdly, it is noticeable that some EST-based molecular markers might be linked to genes involved in controlling traits that are important for market value $[17,33]$. Thus, EST-SSRs can provide a powerful tool for genetic mapping and MAS. In summary, the EST dataset reported here can be used for decoding the molecular mechanism of the flavonoid pathway, as well as lays a foundation for biodiversity, population genetics, genomics, and functional genomics of Epimedium species.

\section{EST-SSR frequency and distribution in Epimedium transcriptome}

A total of 2,810 perfect microsatellites over 12 bp have been identified from Epimedium EST dataset, i.e. 3.67\% EST sequences possess single sequence repeats. Obviously, the frequency of SSRs detected in Epimedium is similar to that of other dicotyledonous species ranging from $2.65 \%$ to $16.82 \%$ [34]. The EST-SSR frequency is dependent on 
the following factors. Firstly, parameters used in exploring microsatellites affect the results dramatically, such as the repeat length threshold and the number of repeat unit. In the present study, mononucleotide repeat motifs are excluded in our strategy for identifying microsatellites. Secondly, the genome structure or composition may also account partially for the EST-SSR frequency, because the genetic-biochemical background of the cells plays important roles in fixation of de novo-generated SSRs [35]. Thirdly, different softwares used in detecting SSRs might also affect the frequency. Some SSR identifying tools can find out imperfect SSR, for example, Sputnik [36], while others can only identify perfect SSRs such as SSRIT [37] and MISA [20,21,26].

Theoretically, the frequency of $\mathrm{di}_{-}$, tri-, tetra-, penta-, and hexanucleotide repeats should be in turn decreased. However, trinucleotide repeat unit is the most dominant SSRs, followed by di-, tetra-, hexa-, and pentanucleotide repeat units (Table 3 ). It is consistent with the results reported for other plant species [20,23,38-40]. As shown in table 4 , the most dominant di- and trinucleotide repeat motifs are AG/CT (19.3\%) and AAG/CTT (23.6\%), respectively. These results are in agreement with that of Morgante et al [36], Kumpatla et al [34], and Toth et al [35]. Interestingly, there are only three CCG/CGG trinucleotide repeats in Epimedium, which is the most predominant in monocots $[36,38]$. Our results strongly support and extend the notion of rarity of the CCG/CGG repeat units in a large number of dicotyledonous plants (Citrus, Coffea, Medicag, Glycine, etc.) [34]. These might be resulted from the high GC content and consequent codon usage bias in monocots [36,38]. Selection might also be against the CCG/CGG repeat unit because of the requirements of the splicing machinery [41]. Long CCG/ CGG sequences could compete splicing machinery components and give rise to inadequate splicing. Moreover, CCG/CGG repeats, might form potential higher structures such as hairpin and quadruplex, and thus affect the efficiency and accuracy of splicing and influence the formation of mature mRNA $[35,42]$.

\section{Primer designing for EST-SSRs}

In this study, attempt of diagnosing transferability have been performed by 32 EST-SSR primer pairs designed according to E. sagittatum ESTs. Unfortunately, seven and four primer pairs fail to transfer in Epimedium and basal eudicot, respectively, which might be due to primer (s) across splice sites, the presence of large introns in genomic sequence, or primer(s) derived from a chimeric or questionable consensuses sequence [39]. Some primer pairs succeed in amplifying in some species while fail in other species, suggesting that null alleles exist. Null alleles might be resulted from some mutations, including the deletion of microsatellites, and indels or substitution in primer binding sites [39]. In addition, the observed sizes of PCR products in Epimedium species deviate frequently from the expected product sizes according to the EST sequences. For instance, polymorphic bands amplified by EsESP05, EsESP25, and EsESP29 are larger than the expected sizes. It might be due to the presence of introns in the corresponding genomic regions $[27,39]$ and/or variation in the repeat numbers.

\section{The EST-SSR transferability in genus Epimedium}

In the present study, transferability of EST-SSRs derived from $E$. sagittatum is high $(85.7 \%)$ across 52 species in genus Epimedium. The high transferability of EST-SSRs is also reported in other plants. For instance, 90\% ESTSSRs in Citrus clementia can be transferred successfully to ten Citrus species and three related genera [20], and 89\% EST-SSRs in Medicago truncatula succeed in transferring to eight genotypes [23]. About 92\% Festuca arundinacea EST-SSR primer pairs can produce characteristic SSR bands in at least one of the six related species [27]. Numerous studies have also demonstrated that EST-SSRs possess higher transferability due to the conservation characteristics of EST-SSRs when compared with SSRs derived from genomic libraries $[43,44]$. In genus Epimedium, the transferability of genomic SSRs derived from $E$. sagittatum [9] and E. koreanum [10] ranges from $21.4 \%$ to $42.9 \%$ and from $42.1 \%$ to $47.4 \%$, respectively. Genomic SSRs isolated from E. brevicornu also possess low transferability ranging from $33.3 \%$ to $66.7 \%$ across other four medicinal Epimedium species [11]. Comparing with the high transferability of EST-SSRs (85.7\%), the transferability of Epimedium genomic SSRs is remarkably lower.

\section{Conclusions}

A large-scale EST dataset with 76,459 consensuses derived from E. sagittatum is reported in this study. A total of 22,295 sequences has been successfully annotated with GO terms based on the known sequences, and part of unique sequences are involved in the flavonoid metabolic pathway, which would facilitate deciphering the molecular mechanism of secondary metabolism in Epimedium. Based on this EST dataset, 2,810 are exploited by MISA software and 32 primer pairs are randomly selected for detecting transferability. The transferability of E. sagittatum EST-SSRs is up to $85.7 \%$ and there are $\sim 1580$ EST-SSR markers transferable in genus Epimedium for further genetic studies. Ho and $\mathrm{He}$ range from 0.04 to 0.6 and from 0.17 to 0.94 with an average of 0.35 and 0.65 , respectively, and the number of alleles per locus ranges from 3 to 27 with an average of 11.9 alleles. These results suggest that the major germplasm of Epimedium show high genetic diversity. Due to the nature of EST-SSRs with high transferability and genomic SSRs with high 
polymorphism and both locating in different regions across whole genomes, combination of EST-SSRs derived from this study and genomic SSRs developed in our lab will be a powerful resource for molecular taxonomic study in genus Epimedium and constructing genetic maps. In addition, EST-SSRs tend to concentrate in the gene-rich regions, suggesting that part of ESTSSRs can be exploited for the use of MAS benefiting for Epimedium molecular breeding in the future. Therefore, the set of EST-SSRs developed in the present study is a promising resource for the related studies of Epimedium. In a word, Epimedium EST dataset is a valuable resource for studies in the fields of taxonomy, MAS, and secondary metabolism in genus Epimedium.

\section{Methods}

\section{Plant materials}

A total of 52 Epimedium species including 55 individuals (Additional file 3) were selected and investigated for the transferability of EST-SSRs derived from $E$. sagittatum. Leaf samples were collected from Wuhan Botanical Garden (Wuhan, Hubei Province, China) and Garden Vision Nursery (Boston, MS, USA). According to the taxa system reported by Stearn [45], samples cover two subgenera and three sections, and represent most of Epimedium germplasm.

\section{cDNA preparation}

The red-magenta fully expanded leaves of E. sagittatum Maxim. grown in Wuhan Botanical Garden, Chinese Academy of Sciences, were harvested and prepared for RNA isolation. Total RNA was isolated according to the instruction of TRIzol kit (Invitrogen) and then purified to exclude the tRNA and rRNA and to enrich mRNA, according to the instruction of mRNA purification kit (Promega). mRNAs were reverse-transcribed by Powerscript $^{\text {tw }}$ II (Takara) with PCR primers SMART IV ${ }^{\text {mo }}$ Oligonucleotide (5'-AAGCAGTGGTATCAACGCAGA GTGGCCATTACGGCCGGG-3') and CDS III/3' PCR Primer (5'-ATTCTAGAGGCCGAGGCGGCCGAC ATG-d(T) $\left.30_{\mathrm{N}-1} \mathrm{~N}-3^{\prime}\right)$. Long Distance PCR for double strand cDNA amplification was performed with LA Taq enzyme (Takara) for 25 cycles $\left(95^{\circ} \mathrm{C}\right.$ for $30 \mathrm{~s}, 68^{\circ} \mathrm{C}$ for 8 min) according to the SMART ${ }^{\mathrm{Tm}}$ cDNA Library Construction Kit User Manual. Finally, double strand cDNAs were purified using DNA purification kit (Qigen) to generate high quality cDNAs.

\section{EST processing, assembly, and annotation}

Around $10 \mu \mathrm{g}$ cDNAs were used for a half-plate sequencing run using 454 GS-FLX pyrosequencing technology. The sequencing was done at the Oklahoma University genome center. Considering the shortness of reads generated by 454 sequencing technology, full-length cDNA was sheared stochastically prior to sequence in order to randomly represent all transcripts. Sequence data for Epimedium Short Read Archive (SRA) described in this paper can be found at the public database [NCBI: SRA008151]. A total of 228,768 raw sequences was obtained. Lucy software [46] with default settings, except that minimum good sequence length cut-off was set to $50 \mathrm{bp}$, was used to remove low quality regions. SeqClean software http://compbio.dfci.harvard.edu/tgi/software/ was then used to eliminate poly $\mathrm{A} / \mathrm{T}$ sequences, vector sequences, adaptor sequences and bacterial sequences. The resulted high quality sequences were assembled to consensus sequences using CAP3 software [47] with parameters as -p 95 -y 15 -s 251 -o 25. All consensus sequences were compared against Pfam database for protein domain analysis [48]. Based on the domain annotation, GO accessions of the consensus sequences were successfully assigned using Pfam 2 go conversion file from the GO consortium http://www.geneontology. org/external2 go. GO annotations were formatted for input into the GOSlim program and the output was parsed to count the occurrence of each GO category.

\section{EST-SSR detection and primer designing}

The EST-SSR detection was performed using Perl program MISA http://pgrc.ipk-gatersleben.de/misa. Since it was hard to distinguish real mononucleotide repeats and single nucleotide stretch error generated by 454 sequencing, mononucleotide repeats were excluded from this study. The parameters were designed for identifying perfect di-, tri-, tetra-, penta-, and hexanucleotide motifs with a minimum of $6,5,4,4$, and 4 repeats, respectively. Primer 3 software [49] was used to design primers. The major parameters for designing the primers were set as follows: primer length ranging from 18 bases to 28 bases with 20 as the optimum, PCR product size ranging from 100 to $300 \mathrm{bp}$, optimum annealing temperature $60^{\circ}$ C, and GC content from $40 \%$ to $70 \%$ with $50 \%$ as optimum. According to these parameters, 32 primer pairs (Additional file 2) were synthesized and were used to detect the transferability in genus Epimedium.

\section{Survey of EST-SSR polymorphism}

Total DNA was isolated from leaf samples according to the CTAB method [50]. PCR amplifications were carried out in a final volume of $10 \mu \mathrm{L}$ containing $50 \mathrm{ng}$ of genomic DNA, $1 \times$ PCR buffer [10 mM Tris- $\mathrm{HCl}(\mathrm{pH} 8.4), 1.5 \mathrm{mM}$ $\mathrm{MgCl}_{2}$ ], $0.2 \mathrm{mM}$ dNTPs, $0.2 \mu \mathrm{M}$ of each primer, and $0.5 \mathrm{U}$ Taq polymerase (Biostar). The PCR reactions were performed under standard conditions for all primers in a thermocycler (Eppendorf). The annealing temperature was fixed for all primer pairs at $60^{\circ} \mathrm{C}$. After $5 \mathrm{~min}$ at $95^{\circ} \mathrm{C}, 35$ cycles were carried out with $30 \mathrm{~s}$ at $95^{\circ} \mathrm{C}, 30 \mathrm{~s}$ at $60^{\circ} \mathrm{C}, 40 \mathrm{~s}$ at $72^{\circ} \mathrm{C}$ for extension, and a final extension step of $10 \mathrm{~min}$ 
at $72^{\circ} \mathrm{C}$. The separation of alleles was performed on a $6 \%$ polyacrylamide gel. PCR products were mixed with an equal volume of loading buffer. The mixture was denatured at $95^{\circ} \mathrm{C}$ for $5 \mathrm{~min}$ before loading onto the gel. Gels were stained with silver nitrate following the protocol as described in Bassam et al [51], and 25 bp DNA marker (Beijing Yuanpinghao Biotech Co., Ltd) was used for calculating the length of EST-SSR amplicons. All primers were detected firstly in E. sagittatum to check if the primers can successful amplify PCR products. Then primers succeeding in amplifying bands were tested for transferability. For detecting whether $E$. sagittatum EST-SSRs transferred successfully or not, the PCR products were firstly tested using $2 \%$ agarose gel and then polyacrylamide gel.

\section{Genetic diversity and data analysis}

Arlequin [52] was used to calculate the genetic diversity and average allele number. Genetic distance of the SSRs genotype was calculated using the log-transformed proportion of shared alleles ('Dps') by MICROSAT [53]. Polymorphic information content (PIC) was calculated by PIC_CALC http://dl.getdropbox.com/u/695591/PIC_CALC.rar combination with GenAlex6 [54]. A dendrogram was constructed based on Neighbor-Joining method [55] in the PHYLIP package [56].

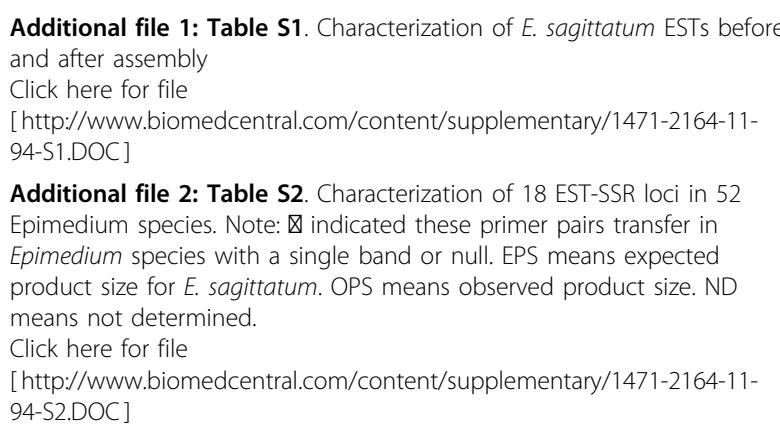

Additional file 3: Table S3. Epimedium species used for detecting the transferability of EST-SSR. Note: E. koreanum1, E. koreanum2, E. koreanum3 indicated that the specimens were collected from Korea, China and Japan, respectively.

Click here for file

[http://www.biomedcentral.com/content/supplementary/1471-2164-1194-S3.DOC ]

Additional file 4: Figure S1. Dendrogram representing the relationships observed in 52 Epimedium species based on 16 EST-SSR markers. $V$. hexandra was used as an outgroup and the distance bar was shown on the bottom of the tree. The number nearby the branch was bootstrap values and the bootstrap value lower than 30\% were not shown. Click here for file

[http://www.biomedcentral.com/content/supplementary/1471-2164-1194-S4.TIFF

Additional file 5: Figure S2. Correlation test between consensus annotation percentage and consensus length. Pearson test show that annotation percentage is positively related to consensus length with correlation coefficients 0.96 at the level of 0.01 .

Click here for file

[http://www.biomedcentral.com/content/supplementary/1471-2164-1194-S5.JPEG ]

\section{Acknowledgements}

This work was partially funded by Knowledge Innovation Project of The Chinese Academy of Sciences (KSCX2-YW-N-030), Presidential Fund of Chinese Academy of Sciences, the CAS/CAFEA International Partnership Program for Creative Research Teams, and 100 Talents Program of Chinese Academy of Sciences. We thank two anonymous reviewers for their kindly suggestions and Dr. Junhua Peng in Wuhan Botanical Garden, Chinese Academy of Sciences, for the help of biological statistics.

\section{Author details}

'Key Laboratory of Pant Germplasm Enhancement and Speciality Agriculture, Wuhan Botanical Garden, Chinese Academy of Sciences, Wuhan, Hubei 430074, China. 'The Graduate School, Chinese Academy of Sciences, Beijing, 100039, China. ${ }^{3}$ Boyce Thompson Institute for Plant Research, Cornell University, Ithaca, NY 14853, USA. ${ }^{4}$ USDA Robert W. Holley Center for Agriculture and Health, Ithaca, NY 14853, USA. ${ }^{5}$ Stephenson Research and Technology Center, University of Oklahoma, Norman, Oklahoma 73019, USA.

\section{Authors' contributions}

SZ prepared transcriptome population for sequencing and developed the EST-SSRs and wrote the manuscript. GX cleaned and assembled the raw sequence and carried out GO annotation. JG performed the genotyping of SSR markers. ZF helped with the 454 sequencing and sequence assembly. 454 sequencing was conducted in BAR's lab. YX helped with the collection of Epimedium plant materials in the field. YW co-designed and assisted in the manuscript preparation. All authors read and approved the final manuscript.

Received: 22 June 2009

Accepted: 8 February 2010 Published: 8 February 2010

\section{References}

1. Committee CP: Herba epimedii. Pharmacopoeia of the People's Republic of China Beijing: Chemical Industry PressCommittee CP 2005, 229-229.

2. Zheng HZ, Dong Z ZH, She Q: Herba epimedii. Modern study of traditional Chinese medicine Beijing: Xueyuan PressZheng HZ, Dong ZH, She Q 1998, 4225-4254.

3. Islam NM, Yoo HH, Lee MW, Dong M, Park Yl, Jeong HS, Kim DH: Simultaneous quantitation of five flavonoid glycosides in Herba epimedii by high-performance liquid chromatography-tandem mass spectrometry. Phytochem Analysis 2008, 19:71-77.

4. Zhang HF, Yang TS, Li ZZ, Wang Y: Simultaneous extraction of epimedin A, B, C and icariin from Herba epimedii by ultrasonic technique. Ultrason Sonochemistry 2008, 15:376-385.

5. Dou JP, Liu ZQ, Liu SY: Structure identification of a prenylflavonol glycoside from Epimedium koreanum by electrospray ionization tandem mass spectrometry. Anal Sci 2006, 22:449-452.

6. Sun Y, Fung KP, Leung PC, Shaw PC: A phylogenetic analysis of Epimedium (Berberidaceae) based on nuclear ribosomal DNA sequences. Mol Phylogenet Evol 2005, 35:287-291.

7. Sun Y, Fung KP, Leung PC, Shi DW, Shaw PC: Characterization of medicinal Epimedium species by $5 \mathrm{~S}$ rRNA gene spacer sequencing. Planta Med 2004, 70:287-288.

8. $\mathrm{Xu} Y, \mathrm{Li} Z$, Wang $Y$, Huang H: Allozyme diversity and population genetic structure of three medicinal Epimedium species from Hubei. J Genet Genomics 2007, 34:56-71.

9. $X u Y Q, L i Z Z$, Wang Y: Fourteen microsatellite loci for the Chinese medicinal plant Epimedium sagittatum and cross-species application in other medicinal species. Mol Ecol Res 2008, 8:640-642.

10. Zhou JF, Xu YQ, Huang HW, Wang Y: Identification of microsatellite loci from Epimedium koreanum and cross-species amplification in four species of Epimedium (Berberidaceae). Mol Ecol Notes 2007, 7:467-470.

11. Xu Y, Huang $H, L i Z$, Wang Y: Development of 12 novel microsatellite loci in a traditional Chinese medicinal plant, Epimedium brevicornum and cross-amplification in other related taxa. Conserv Genet 2008, 9:949-952.

12. Nakai R, Shoyama Y, Shiraishi S: Genetic characterization of Epimedium species using random amplified polymorphic DNA (RAPD) and PCR- 
restriction fragment length polymorphism (RFLP) diagnosis. Biol Pharm Bull 1996, 19:67-70.

13. Shen P, Guo BL, Gong Y, Hong DYQ, Hong Y, Yong EL: Taxonomic, genetic, chemical and estrogenic characteristics of Epimedium species. Phytochemistry 2007, 68:1448-1458.

14. Zhang ML, Uhink CH, Kadereit JW: Phylogeny and biogeography of Epimedium/Nancouveria (Berberidaceae): Western North American - East Asian disjunctions, the origin of European mountain plant taxa, and East Asian species diversity. Syst Bot 2007, 32:81-92.

15. Jiang T, Li Y, Yang Z, Han S, Chen G: Construction and identification of a CDNA library for the flower buds of Epimedium brevicornu Maxim. $J$ Wuhan Bot Res 2007, 25:105-108.

16. Qian B, Li Y, Yang Z, Feng T: Construction of CDNA library in Epimedium brevicornum leaves. Guihaia 2007, 27:518-521.

17. Bozhko M, Riegel R, Schubert R, Muller-Starck G: A cyclophilin gene marker confirming geographical differentiation of Norway spruce populations and indicating viability response on excess soil-born salinity. Mol Ecol 2003, 12:3147-3155.

18. Scott KD, Eggler P, Seaton G, Rossetto M, Ablett EM, Lee LS, Henry RJ: Analysis of SSRs derived from grape ESTs. Theor Appl Genet 2000, 100:723-726.

19. Yu JK, La Rota M, Kantety RV, Sorrells ME: EST derived SSR markers for comparative mapping in wheat and rice. Mol Genet Genomics 2004, 271:742-751.

20. Luro FL, Costantino G, Terol J, Argout X, Allario T, Wincker P, Talon M, Ollitrault P, Morillon R: Transferability of the EST-SSRs developed on Nules clementine (Citrus clementina Hort ex Tan) to other Citrus species and their effectiveness for genetic mapping. BMC Genomics 2008, 9:287.

21. Aggarwal RK, Hendre PS, Varshney RK, Bhat PR, Krishnakumar V, Singh L: Identification, characterization and utilization of EST-derived genic microsatellite markers for genome analyses of coffee and related species. Theor Appl Genet 2007, 114:359-372.

22. Poncet V, Rondeau M, Tranchant C, Cayrel A, Hamon S, de Kochko A, Hamon P: SSR mining in coffee tree EST databases: potential use of ESTSSRs as markers for the Coffea genus. Mol Genet Genomics 2006, 276:436-449.

23. Eujayl I, Sledge MK, Wang L, May GD, Chekhovskiy K, Zwonitzer JC, Mian MAR: Medicago truncatula EST-SSRs reveal cross-species genetic markers for Medicago spp. Theor Appl Genet 2004, 108:414-422.

24. Peakall R, Gilmore S, Keys W, Morgante M, Rafalski A: Cross-species amplification of soybean (Glycine max) simple sequence repeats (SSRs) within the genus and other legume genera: Implications for the transferability of SSRs in plants. Mol Biol Evol 1998, 15:1275-1287.

25. Gaitan-Solis E, Duque MC, Edwards KJ, Tohme J: Microsatellite repeats in common bean (Phaseolus vulgaris): isolation, characterization, and crossspecies amplification in Phaseolus ssp. Crop Sci 2002, 42:2128-2136.

26. Thiel T, Michalek W, Varshney RK, Graner A: Exploiting EST databases for the development and characterization of gene-derived SSR-markers in barley (Hordeum vulgare L.). Theor Appl Genet 2003, 106:411-422.

27. Saha MC, Mian MAR, Eujayl I, Zwonitzer JC, Wang LJ, May GD: Tall fescue EST-SSR markers with transferability across several grass species. Theor Appl Genet 2004, 109:783-791.

28. Zhang $L Y$, Bernard $M$, Leroy $P$, Feuillet $C$, Sourdille P: High transferability of bread wheat EST-derived SSRs to other cereals. Theor Appl Genet 2005, 111:677-687.

29. Zhao HY, Sun JH, Fan MX, Fan L, Zhou L, Li Z, Han J, Wang BR, Guo DA: Analysis of phenolic compounds in Epimedium plants using liquid chromatography coupled with electrospray ionization mass spectrometry. J Chromatogr A 2008, 1190:157-181.

30. Wang W, Chen ZD, Liu Y, Li RQ, Li JH: Phylogenetic and biogeographic diversification of Berberidaceae in the northern hemisphere. Syst Bot 2007, 32:731-742.

31. Margulies M, Egholm M, Altman WE, Attiya S, Bader JS, Bemben LA, Berka J, Braverman MS, Chen Y, Chen Z, et al: Genome sequencing in microfabricated high-density picolitre reactors. Nature 2005, 437:376-380.

32. Emrich SJ, Barbazuk WB, Li L, Schnable PS: Gene discovery and annotation using LCM-454 transcriptome sequencing. Genome Res 2007, 17:69-73.

33. Schubert R, Mueller-Starck G, Riegel R: Development of EST-PCR markers and monitoring their intrapopulational genetic variation in Picea abies (L.) Karst. Theor Appl Genet 2001, 103:1223-1231.
34. Kumpatla SP, Mukhopadhyay S: Mining and survey of simple sequence repeats in expressed sequence tags of dicotyledonous species. Genome 2005, 48:985-998.

35. Toth $G$, Gaspari Z, Jurka J: Microsatellites in different eukaryotic genomes: survey and analysis. Genome Res 2000, 10:967-981.

36. Morgante $M$, Hanafey $M$, Powell W: Microsatellites are preferentially associated with nonrepetitive DNA in plant genomes. Nat Genet 2002, 30:194-200.

37. Kantety R, La Rota M, Matthews D, Sorrells M: Data mining for simple sequence repeats in expressed sequence tags from barley, maize, rice, sorghum and wheat. Plant Mol Biol 2002, 48:501-510.

38. La Rota M, Kantety RV, Yu JK, Sorrells ME: Nonrandom distribution and frequencies of genomic and EST-derived microsatellite markers in rice, wheat, and barley. BMC Genomics 2005, 6:23.

39. Varshney RK, Graner A, Sorrells ME: Genic microsatellite markers in plants: features and applications. Trends Biotech 2005, 23:48-55.

40. Varshney RK, Thiel T, Stein N, Langridge P, Graner A: In silico analysis on frequency and distribution of microsatellites in ESTs of some cereal species. Cell Mol Biol Lett 2002, 7:537-546.

41. Li YC, Korol AB, Fahima T, Nevo E: Microsatellites within genes: structure, function, and evolution. Mol Biol Evol 2004, 21:991-1007.

42. Coleman TP, Roesser JR: RNA secondary structure: an important ciselement in rat calcitonin/CGRP pre-messenger RNA splicing. Biochemistry 1998, 37:15941-15950.

43. Cho YG, Ishii T, Temnykh S, Chen X, Lipovich L, McCouch SR, Park WD, Ayres N, Cartinhour S: Diversity of microsatellites derived from genomic libraries and GenBank sequences in rice (Oryza sativa L.). Theor Appl Genet 2000, 100:713-722.

44. Eujayl I, Sorrells ME, Baum M, Wolters P, Powell W: Isolation of EST-derived microsatellite markers for genotyping the $A$ and $B$ genomes of wheat. Theor Appl Genet 2002, 104:399-407.

45. Stearn WT: Taxonomic treatment of Epimedium. The genus Epimedium and other herbaceous Berberidaceae Oregon: Timber PressStearn WT 2002, 41-199.

46. Chou HH, Holmes MH: DNA sequence quality trimming and vector removal. Bioinformatics 2001, 17:1093-1104.

47. Huang X, Madan A: CAP3: A DNA Sequence Assembly Program. Genome Res 1999, 9:868-877.

48. Bateman A, Birney E, Durbin R, Eddy SR, Howe KL, Sonnhammer ELL: The Pfam protein families database. Nucl Acids Res 2000, 28:263-266.

49. Rozen S, Skaletsky H: Primer3 on the WWW for general users and for biologist programmers. Methods Mol Biol 2000, 132:365-386.

50. Porebski S, Bailey LG, Baum BR: Modification of a CTAB DNA extraction protocol for plants containing high polysaccharide and polyphenol components. Plant Mol Biol Rep 1997, 15:8-15.

51. Bassam BJ, Caetanoanolles G, Gresshoff PM: Fast and sensitive silver staining of DNA in polyacrylamide gels. Anal Biochem 1991, 196:80-83.

52. Excoffier L, Laval G, Schneider S: Arlequin version 3.0: an integrated software package for population genetics data analysis. Evol Bioinform Online 2005, 1:47-50.

53. MICROSAT: a computer program for calculating various statistics on microsatellite allele data, version $1.5 \mathrm{c}$. http://hpgl.stanford.edu/projects/ microsat/.

54. Peakall R, Smouse PE: GENALEX 6: genetic analysis in Excel. Population genetic software for teaching and research. Mol Ecol Notes 2006, 6:288-295.

55. Saitou N, Nei M: The Neighbor-Joining method: A new method for reconstructing phylogenetic trees. Mol Biol Evol 1987, 4:406-425.

56. Felsenstein J: PHYLIP (Phylogeny Inference Package) version 3.6. Distributed by the author Department of Genome Sciences, University of Washington, Seattle 2004.

doi:10.1186/1471-2164-11-94

Cite this article as: Zeng et al: Development of a EST dataset and

characterization of EST-SSRs in a traditional Chinese medicinal plant, Epimedium sagittatum (Sieb. Et Zucc.) Maxim. BMC Genomics 2010 11:94. 